\title{
Empleo de residuos industriales siderúrgicos como materiales aglomerantes en construcción
}

\author{
V.A. Mymrin $\left.{ }^{*}\right)$
}

\begin{abstract}
Resumen Como materiales aglomerantes, se pueden utilizar diferentes tipos de residuos, tales como escorias, residuos sólidos y líquidos alcalinos procedentes de la industria siderúrgica, así como otras escorias procedentes de los sectores no férreos. Su empleo como nuevos materiales, tales como hormigones pobres, destinados a aplicaciones tales como bases de carreteras, aeropuertos, suelos industriales y presas en sustitución de piedra triturada, arena y grava. Todos estos residuos industriales, perjudiciales para el medio ambiente desde el punto de vista ecológico, se pueden transformar en nuevos materiales estables en los que no ocurre la lixiviación de los materiales pesados que contienen. Los nuevos materiales resultan de la combinación, con la dosificación adecuada, de dos o tres de los residuos industriales mencionados o de la mezcla de algunos con suelos naturales. En la producción de estos nuevos materiales que adquieren una nueva estructura distinta de sus componentes, no se emplean ligantes tradicionales, como el cemento, ni es necesario su calentamiento. A las ventajas de la eliminación de estos residuos industriales contaminantes, se añaden otras de tipo económico, ya que pueden obtenerse reducciones de coste de hasta 5 ó 6 veces. Se presentan ejemplos de aplicaciones reales en carreteras en diferentes partes de Rusia, incluida Siberia, y otras zonas del norte, cuya vida en servicio supera los 20 años y que todavía se encuentran en un perfecto estado de conservación.
\end{abstract}

Palabras clave: Residuos industriales. Escoria férrea de escombreras. Materiales aglomerantes. Transición sol gel. Construcción de carreteras.

\section{Use of ferrous industrial wastes as binding materials for construction}

\begin{abstract}
Several ferrous and non ferrous metallurgical wastes, slags, powdered wastes, alcaline liquors, etc., can be used as binding materials to produce new building materials. These materials can be used in place of concretes made of cement, crushed stones, sand and gravel mixtures in several applications, road, industrial and airport foundations, etc. They are leaching resistant, so heavy metals do not migrate to the environment. These new materials, with a new structure, are obtained by mixing in right proportion of two or three industrial wastes or with mixtures of two of them and natural soils without any addition of traditional binders like cement and without heating. The main advantages are the solution of the problem of disposal of industrial wastes and the lower cost of the new materials, 5 to 6 times cheaper than traditional. Several examples of roads existing in Russia, even in Siberia and other northern regions, demonstrate the benefit of this process because after 20 years they still offer a good performance.
\end{abstract}

Keywords: Industrial wastes. Ferrous dumped slags. Binding materials. Sol-gel transition. Road construction

\section{INTRODUCCIÓN}

La gran dimensión de la industria metalúrgica, y en particular de la industria siderúrgica, convierte el

(*) Centro Nacional de Investigaciones Metalúrgicas, CENIM (CSIC), Avda. de Gregorio del Amo, 8. 28040-Madrid (España) e Instituto de Geociencias Medioambientales (RAN). Moscú (Rusia). problema de la eliminación de los productos residuales de sus diferentes procesos en un problema grave dado su tonelaje y el contenido en diferentes metales pesados, cuya transferencia al medio ambiente se debe evitar reduciéndola por debajo de los límites de las actuales regulaciones comunitarias.

Cualquier procesamiento buscando su inertización implica un coste más o menos elevado, por lo 
que la estrategia del tratamiento de estos materiales ha experimentado una evolución en el sentido de que se intenta su conversión en otros que puedan tener aplicaciones que contribuyan a disminuir los costes del procesamiento, los compense o, en el caso más favorable, lleguen a producir algún tipo de beneficio.

La línea tradicional seguida por diversos autores (1 y 2) busca la inertización de estos materiales como sustitutos de la piedra machacada, sin aprovechar sus propiedades reactivas como ligante, porque se cree que la exposición al aire en las escombreras le han hecho perder estas características.

En este trabajo, se resumen los resultados obtenidos a lo largo de 20 años y de los que se encuentran numerosas aplicaciones reales en Rusia, incluida Siberia y otras zonas en las que las condiciones climatológicas extremas revalorizan la calidad del material que se obtiene (3 y 4). Estos resultados demuestran la posibilidad de la activación mecánica y química de las propiedades aglomerantes de las escorias siderúrgicas de escombreras.

Las calidades ligantes de alguno de los residuos empleados en la fabricación de estos nuevos materiales deben reunir unas características que los hagan susceptibles de utilización en diversos campos de aplicación y que eviten cualquier riesgo de difusión de los metales pesados al entorno medioambiental.

Los procesos de obtención de estos materiales han sido objeto de diversas patentes (5-8). De modo general, se puede señalar que, como materiales aglomerantes, se pueden usar distintos tipos de escorias de la industria metalúrgica, férrea o no férrea, así como residuos de industrias de mecanizado o de fundición de hierro (escorias y arenas quemadas).

Como aditivos que producen el refuerzo se usaron distintos tipos de residuos industriales, tales como residuos de minas de carbón quemados, escorias y cenizas de combustión de plantas térmicas que emplean antracitas y carbones de menor calidad eliminados por vía húmeda, restos de industrias de corte de piedras, etc.

Además de estos productos, se pueden emplear, como elementos de refuerzo, diversos tipos de suelos naturales, como los utilizados en la anterior Unión Soviética, procedentes de zonas de diversa geografía, geomorfología, geobotánica y clima.

Como activadores de las mezclas se emplearon residuos secos o licores procedentes de muy diversas plantas (químicas, petroquímicas, metal-mecánicas, metalúrgicas, térmicas, textiles, de barnices y pinturas, etc.). Estos aditivos son los encargados de realizar la interacción química de los materiales de unión y refuerzo utilizados.

\section{TRABAJO REALIZADO}

La amplia diversidad de estos residuos, susceptibles de usarse como materias primas en la producción de estos nuevos materiales, exige un control de sus características para conseguir las mezclas adecuadas que tengan las propiedades deseadas. Estas características, sin embargo, serán diferentes de un país a otro, porque los procesos y las regulaciones medioambientales son también muy diferentes. Esto produce la necesidad de reestudiar los problemas in situ en el caso de contextos legales diferentes.

Así, en Rusia, el contenido de $\mathrm{FeO}+\mathrm{Fe}_{2} \mathrm{O}_{3}$ en las escorias es muy elevado, llegando a ser del orden del 22 al $32 \%$. El contenido de $\mathrm{CaO}$ en las escorias de convertidor de horno alto y de fundición es también elevado (45-56\%). El contenido de $\mathrm{SiO}_{2}$ alcanza sus valores más altos en el caso de las arenas de fundición (87-92\%).

Sin embargo, los análisis petrográficos y por rayos $\mathrm{X}$ para la identificación de fases ponen de manifiesto que estas diferencias son mucho mayores que las de su composición química. Así, por ejemplo, en las escorias de horno alto se puede encontrar melilita (25-30\%), silicato bicálcico beta $(10 \%)$, pseudowollastonita (5\%) y fase vítrea $(60-70 \%)$, composiciones que pueden ser muy diferentes en escorias de otra procedencia. Las mayores semejanzas se encuentran, sin embargo, en las escorias de horno eléctrico y de reverbero, aunque aquí las composiciones químicas pueden ser muy diferentes entre sí.

El componente más próximo al cemento Portland lo constituyen las escorias de convertidor, que incluyen minerales, tales como la alita $(45 \%)$ y belita $(20 \%)$, que permiten esperar de ellas la máxima capacidad como ligantes.

De modo general, cabe señalar que la humedad a la que se lleva a cabo el proceso (10-12\%) constituye un parámetro clave y que se debe aplicar una cierta presión para favorecer el contacto íntimo entre los residuos (1 MPa) durante un tiempo relativamente breve $(1 \mathrm{~min})$. El endurecimiento tiene que llevarse a cabo en condiciones de humedad del orden del $98 \%$.

\section{PRESENTACIÓN DE RESULTADOS}

\subsection{Endurecimiento de residuos industriales como materiales de unión}

Como se ha indicado, las propiedades de los materiales depende de la composición química de los componentes y de sus proporciones (Tabla I, ítems 1-8). La resistencia de los materiales tras 90 días de 
TABLA I.- Propiedades mecánicas de las escorias férreas activadas

TABLE I.-Mechanical properties of activated ferrous slags

\begin{tabular}{|c|c|c|c|c|c|c|c|c|c|c|}
\hline \multicolumn{2}{|c|}{ Material de unión } & \multicolumn{5}{|c|}{ Resistencia a la compresión ( $\mathrm{MPa}$ ) } & \multicolumn{4}{|c|}{ Resistencia al agua y al frío } \\
\hline Item & Ligante & $\begin{array}{c}28 \\
\text { días }\end{array}$ & $\begin{array}{c}90 \\
\text { días }\end{array}$ & $\begin{array}{c}1 \\
\text { año }\end{array}$ & $\begin{array}{c}4 \\
\text { años }\end{array}$ & $\begin{array}{c}6 \\
\text { años }\end{array}$ & $\begin{array}{c}R_{a} \\
\mathrm{MPa}\end{array}$ & $C_{a}$ & $\begin{array}{c}R_{f} \\
\mathrm{MPa}\end{array}$ & $C_{f}$ \\
\hline 1 & \multirow{2}{*}{ Horno alto } & 3,4 & 5,8 & 17,2 & 18,0 & 18,9 & 6,2 & 1,1 & 5,1 & 0,8 \\
\hline 2 & & 0,9 & 5,1 & 14,1 & 12,2 & 15,4 & 4,8 & 0,9 & 4,6 & 1,0 \\
\hline 3 & \multirow{2}{*}{ H. reverbero } & 1,6 & 2,4 & 11,1 & 13,8 & 19,4 & 2,7 & 1,2 & 2,6 & $\overline{1,0}$ \\
\hline 4 & & 0,8 & 2,5 & 4,6 & 11,9 & 14,4 & 2,6 & 1,1 & 2,1 & 0,8 \\
\hline 5 & \multirow{2}{*}{ H. eléctrico } & 3,1 & 2,2 & 2,8 & 13,6 & 16,4 & 2,6 & 1,2 & 1,2 & 0,5 \\
\hline 6 & & 3,9 & 7,3 & 10,6 & 7,5 & 9,6 & 7,7 & 1,1 & 6,0 & 0,8 \\
\hline 7 & \multirow{2}{*}{ Convertidor } & 1,6 & 11,6 & 17,9 & 39,9 & - & 12,2 & 1,1 & 9,0 & 0,7 \\
\hline 8 & & 2,4 & 7,0 & 32,1 & 47,6 & - & 3,9 & 0,6 & 5,7 & 1,5 \\
\hline
\end{tabular}

$R_{a}$ y $R_{f}$. Resistencia al agua de las probetas después de 25 ciclos de enfriamiento y secado $\left(-25^{\circ} \mathrm{C}\right.$ a $\left.+20^{\circ} \mathrm{C}\right)$. $C_{a}$ y $C_{f}$. Coeficientes de resistencia al agua y al frío de las probetas.

envejecimiento osciló entre 2,2 y 11,6 MPa y aumentó al cabo de 1 año a 2,8-32,1 MPa, alcanzando valores de 7,5 a 47,6 al cabo de 4 años. Los productos más resistentes se obtuvieron como resultado de la activación de las escorias de convertidor (ítems 7 y 8 ) en los que el contenido en $\mathrm{CaO}$ era máximo.

El coeficiente $C_{a}$ viene dado por la fórmula $C_{a}=$ $R_{a} / R_{a h}$, donde $R_{a}$ es su resistencia a la compresión tras 24 h en agua y $R_{a h}$ es la resistencia a la compresión, tras mantener las probetas en aire saturado en vapor de agua, y el coeficiente $C_{f}$ viene dado por la fórmula $C_{f}=R_{f} / R_{a}$, donde $R_{f}$ es la resistencia a la compresión de las probetas después de 25 ciclos de enfriamiento a $-25{ }^{\circ} \mathrm{C}$ e inmersión en agua a $+25^{\circ} \mathrm{C}$.

La investigación por rayos $\mathrm{X}$ de los materiales puso de manifiesto que las muestras no presentaban ningún crecimiento de nuevas formas cristalinas tras 20 años de vida en servicio, que serían las responsables del aumento de su resistencia. Este incremento se debe al crecimiento de un amorfo tipo gel. En las escorias con exceso de fase vítrea y carentes de minerales activos del cemento Portland necesarios para el lixiviado del vidrio, el proceso de cristalización del gel no se manifiesta, incluso después de 20 años.

La superficie de las partículas de escoria son lixiviadas y se forma nuevos productos amorfos en el medio alcalino cuando se hidrata la escoria, según se comprobó mediante análisis con rayos X (Fig. 1). La cantidad de cristales formados, sobre todo carbonatos, es insignificante durante la hidratación de la escoria. Aparentemente, el proceso de endurecimiento de los materiales se debe a la formación y crecimiento de compuestos amorfos en forma de gel, que rellenan los huecos del volumen de las muestras actuando como ligante entre los granos de

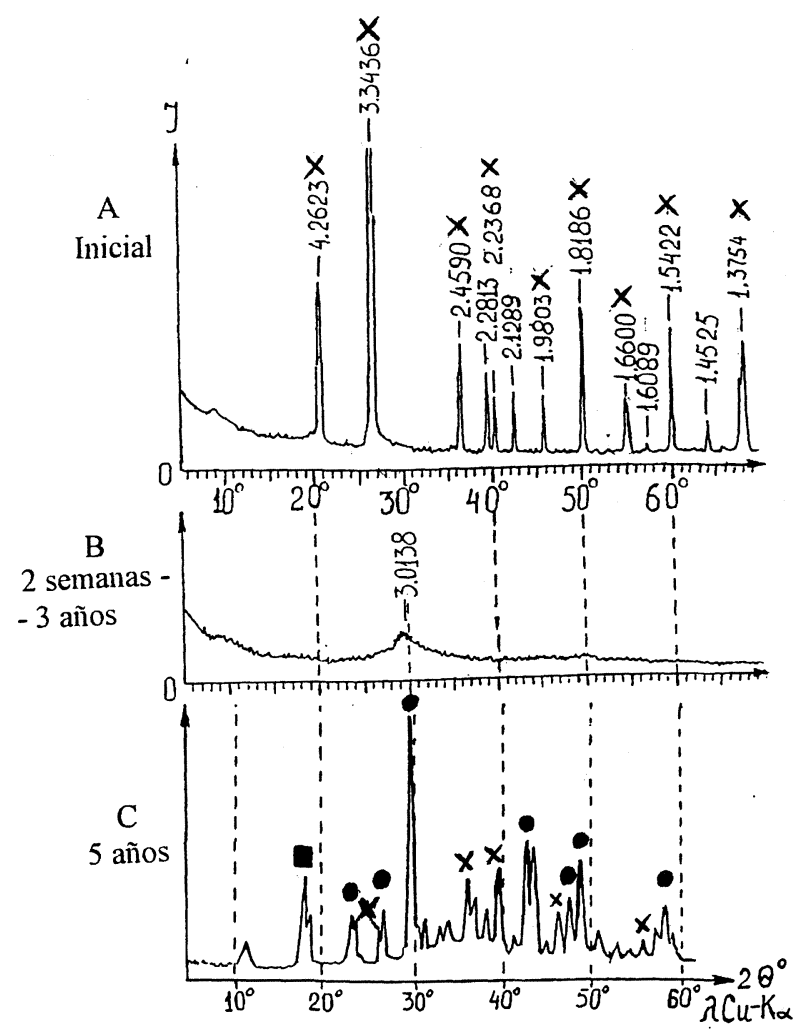

Fig. 1.- Difractogramas de rayos X de $A$ ) Mezcla inicial de arena de fundición, escorias de escombreras y líquido activador (residuos industriales). $B$ ) $\mathrm{La}$ misma mezcla después de 2 semanas a 3 años, conservada al aire libre. $C$ ) La misma mezcla después de 5 años, conservada la aire libre. $x=$ Cuarzo $\alpha$. $\bullet=$ Calcita. $=$ Portlandita.

FIG. 1.- X Ray difractogram. A) Iron foundry sand, dump slag and activator solution (industrial waste). B) After 2 weeks to 3 years in open air. C) After 5 years. $\times=\alpha$-Quartz. $\cdot=$ Calcite $\cdot=$ Portlandite . 
TABLA II.- Propiedades mecánicas de las composiciones de mezcla escoria-suelo-activador recomendadas para bases de carretera

TABLE II.-Mechanical properties of mixture composition slag-soil-activator recommeded as road bases

\begin{tabular}{|c|c|c|c|c|c|c|c|c|c|}
\hline \multicolumn{3}{|c|}{ Composición de los materiales } & \multicolumn{3}{|c|}{$\begin{array}{l}\text { Resistencia a la } \\
\text { compresión, (MPa) }\end{array}$} & \multicolumn{4}{|c|}{ Resistencia al agua y al frío } \\
\hline Item & Suelos & Ligantes & $\begin{array}{c}28 \\
\text { días }\end{array}$ & $\begin{array}{c}90 \\
\text { días }\end{array}$ & $\begin{array}{l}360 \\
\text { días }\end{array}$ & $\begin{array}{c}R_{a} \\
\mathrm{MPa}\end{array}$ & $C_{a}$ & $\begin{array}{c}R_{f} \\
\mathrm{MPa}\end{array}$ & $C_{f}$ \\
\hline 1 & Marga ligera & Horno alto & 2,89 & 2,58 & 3,63 & 2,48 & 0,96 & 2,08 & 0,84 \\
\hline 2 & Marga pesada & Id & 2,52 & 3,13 & 6,04 & 2,91 & 0,96 & 2,30 & 0,79 \\
\hline 3 & Arena media & H. reverbero & 1,47 & 2,96 & 5,61 & 3,37 & 1,04 & 4,08 & 1,16 \\
\hline 4 & Marga ligera & Id & 1,14 & 1,87 & 6,96 & 1,74 & 0,93 & 1,50 & 0,86 \\
\hline 5 & Marga tipo loes & H. eléctrico & 4,83 & 9,24 & 7,84 & 9,57 & 1,16 & 7,96 & 0,83 \\
\hline 6 & Marga tipo loes & Id & 5,77 & 11,9 & 14,43 & 13,6 & 1,13 & 11,0 & 0,81 \\
\hline 7 & Marga pesada & Convertidor & 2,13 & 3,74 & 8,59 & 4,26 & 1,14 & 3,32 & 0,78 \\
\hline 8 & $\begin{array}{l}\text { Arena de fundición } \\
\text { quemada }\end{array}$ & Escoria de fundición & $\begin{array}{l}1,14- \\
2,28\end{array}$ & $\begin{array}{l}5,00- \\
6,21\end{array}$ & $\begin{array}{r}9,83- \\
13,40\end{array}$ & $\begin{array}{l}5,58- \\
6,33\end{array}$ & $\begin{array}{l}0,94- \\
1,14\end{array}$ & $\begin{array}{l}4,83- \\
7,09\end{array}$ & $\begin{array}{l}0,84- \\
1,27\end{array}$ \\
\hline 9 & $\begin{array}{l}\text { Rechazos de mina } \\
\text { de carbón }\end{array}$ & $\begin{array}{l}\text { Mezcla de escorias de } \\
\text { horno alto y } \\
\text { fundición de acero }\end{array}$ & $\begin{array}{l}1,78- \\
0,56\end{array}$ & $\begin{array}{l}2,97- \\
1,67\end{array}$ & $\begin{array}{l}3,20- \\
1,89\end{array}$ & $\begin{array}{l}2,50- \\
1,34\end{array}$ & $\begin{array}{l}1,16- \\
0,78\end{array}$ & $\begin{array}{l}2,05- \\
0,96\end{array}$ & $\begin{array}{l}1,87- \\
0,72\end{array}$ \\
\hline
\end{tabular}

$R_{a}$ y $R_{f}=$ Resistencia al agua de las probetas tras 25 ciclos de enfriamiento a $-25^{\circ} \mathrm{C}$ e inmersión a $+20^{\circ} \mathrm{C}$. $C_{a}$ y $C_{f}$. Coeficientes de resistencia al agua y al frío.

cristales y produciendo una estructura de coagulación cristalina.

\subsection{Endurecimiento con suelos naturales o artificiales con estos residuos férreos}

Se ha demostrado experimentalmente que las escorias, después de activación mecánica por molido y activación química por adición de otros residuos industriales, resultan unos ligantes muy eficaces para la estabilización del suelo (Tabla II).

Los suelos naturales se usan como base para su estabilización cuando no se dispone de suelos artificiales. Sus propiedades finales dependen, naturalmente, de sus características específicas y de las proporciones en que se mezclan (Tabla II, ítems 19). Las resistencias obtenidas a 90 días oscila entre 1,87 y 11,98; a 1 año alcanzan valores que van de 3,63 a 14,43 . Estos productos son muy resistentes a la lixiviación y al frío.

Los mejores resultados obtenidos se consiguieron con las mezclas entre los loes de Uzbekistán y las escorias de horno eléctrico. Los loes tienen elevados porcentajes de cloruros y sulfatos. Los minerales carbonatados también son un material beneficioso.
Si observamos los datos del ítem 8 (Tabla II) vemos la utilidad de las mezclas de escoria de fundición y arenas quemadas como material para bases de carreteras y otras aplicaciones. Después de 90 días, su resistencia fue de 5-6,2 MPa, que es el máximo de los requisitos exigidos en las normas rusas para suelos reforzados. Estos valores se duplican al cabo de 1 año y los materiales son muy resistentes a la lixiviación y al frío con coeficientes que superan la unidad.

En las mezclas de escoria de fundición-arena-activador, empleadas en Siberia y en diversas zonas próximas al Volga, se vio que, durante el proceso de endurecimiento, tiene lugar la total disolución de las partículas de los residuos de fundición y de todos los cristales en la solución dentro de los poros. Se vio que la existencia de proporciones crecientes de humus tenía un efecto perjudicial porque reducían la resistencia.

Se comprobó que las mezclas de escoria de fundición-arena-activador debían el incremento de su resistencia a la transformación que ocurría en el interior de los poros, donde la solución pasaba de la forma sol a la forma gel, como resultado de numerosas etapas de la sinéresis del gel. El posterior incremento de la densidad de la solución facilita su transformación en gel. El endurecimiento del gel se 
inicia con su sinéresis y son estas numerosas etapas las que transforman la mezcla en un estado pétreo.

De este modo, se consigue que sustancias química y mineralógicamente muy complicadas, como las que se han descrito en este trabajo, al disolverse puedan formar una estructura amorfa, de acuerdo con la ley cristaloquímica de Grot-Fedorov, que establece que la sustancia químicamente más compleja tiene un menor hábito cristalino de su estructura mineral, como muchos minerales naturales (hisingerita, limonita, etc.) o rocas sedimentarias (flint, opoka, trepolita, etc.). Estos minerales naturales y rocas se han mantenido durante períodos geológicos sin transformación de su estructura amorfa en cristalina.

Evidentemente, en el medio donde se encuentran las escorias activadas por el medio alcalino tienen lugar reacciones intensivas de formación de compuestos por quimisorción o partículas de suelos arcillosos con poros cerrados. El proceso de cierre de poros termina tras 60-90 días. No obstante, el proceso de endurecimiento continúa a lo largo de varios años.

\section{CONCLUSIONES}

- A partir de consideraciones teóricas universalmente admitidas, se demuestra, de modo experimental, que mezclas adecuadas, desde un punto de vista químico y mineralógico, de diversos residuos industriales metalúrgicos (escorias férreas y de fundición) aseguran las características de ligantes de los residuos, que se pueden incrementar mediante trituración mecánica y adición de otros residuos industriales que permitan la estabilización de suelos naturales o artificiales.

- El empleo de estos materiales reduce los problemas del tratamiento de los residuos de los que se obtiene, con unas tasas de encarecimiento previsibles muy elevadas, valoriza el empleo de los suelos y permite disminuir el consumo de materiales naturales, arena, roca, grava, etc., evitando la alteración del medio ambiente por extracción de arenas y canteras, y los costes de transporte.
El coste final del producto puede llegar a ser 5-6 veces menor que la alternativa actual.

- El incremento de la resistencia de las mezclas escoria-suelo-activador se debe a la total disolución de las partículas sólidas y al crecimiento de compuestos de gel amorfos y su sinéresis y transición al estado pétreo por un proceso sol-gel.

\section{Agradecimiento}

Al CSIC por su concesión de un año sabático y a la Profesora. $\mathrm{M}^{\mathrm{a}}$ del Carmen Andrade Perdrix, Directora del IETCC, y al Prof. Alfonso J. Vázquez, del CENIM, por su ayuda experimental y apoyo personal en la verificación de este trabajo empleando los residuos industriales que se producen en España, que el autor está llevando a cabo en los laboratorios del Departamento de Corrosión y Protección del CENIM.

\section{REFERENCIAS}

(1) LEE, A.R. Blast furnace and steel slag. Production, properties and uses. Londres (R.U.) Edward Arnold Publ. Ltd. 1974: 119.

(2) Information Day on the utilization of blast furnace and steelmaking slags. Proc.Conf. ECSC Lieja. Bruselas (Bélgica) 1998.

(3) Mymrin, V.A. 1994. Solid and liquid industrial wastes as compounds of new construction materials. Proc. III Intern. Cong. Chemistry of ANQUE, p. 108. Islas Canarias (España).

(4) Mymrin, V.A. Quím. Ind. 41 (2), 1994: 106-110.

(5) Mymrin, V.A. 1990. Method of construction of road bases and foundations. Pat. rusa núm. 2028408, Bulletin No. 4, 1995 Moscú (Rusia).

(6) Mymrin, V.A. y Kochetkova, P.G. 1992. Method of construction of road bases and foundations. Pat. rusa núm. 2030507. Bulletin No 7, 1995. Moscú (Rusia).

(7) Mymrin, V.A. 1991. Method of construction of road bases and foundations. Pat. rusa núm.14, 1996, Moscú (Rusia).

(8) Mymrin, V.A. 1996. Composition for construction of road bases and foundation positive decision on the Russian patents distribution for the claim No 96111210, Moscú (Rusia). 
PROGRAMA DEL $8^{\circ}$ CONGRESO DE CIENCIA Y TECNOLOGÍA METALÚRGICAS 26 A 29 DE MAYO DE 1998

\section{MARTES 26}

Inscripción: 15:00 - 19:00 h

Visita a Industria TRATERH: salida del autobús a las 16 h del Hotel Meliá.

Recepción y vino español ofrecido por el Ayuntamiento de Pinto.

\section{MIÉRCOLES 27}

Inscripción: 8:00 - 9:15 h

9:15 - 9:30 h: INAUGURACIÓN a cargo del Presidente del CSIC, César Nombela.

9:30 - 10:30 h: CONFERENCIA PLENARIA a cargo de Arturo García Arroyo (DG XII, UE): "La investigación europea siderúrgica en el V Programa Marco". Presidencia: César Nombela (CSIC) y Carlos Avello (ACERALIA).

10:30 - 12:00 h: Café y sesión de paneles y exposición

12:00 -14:00 h: Sesión 1 de conferencias (de 15+5 min): METALURGIA FISICA. Presidente: Ignacio Les (SIDENOR). Secretario: Carlos García de Andrés (CENIM).

1) 12:00-12:20: V.F. Rodríguez, J.A. Jiménez, P. Adeva, A. Bohórquez, G.A. Pérez, B.J. Fernández y J. Chao. Propiedades mecánicas y mecanismos de deformación en aleaciones del sistema Fe-xMn-3,2Al$0,2 \mathrm{C}(12 \times 43)$.

2) 12:20-12:40: J.M. Cabrera y J.M. Prado. Ecuaciones constitutivas de la fluencia en caliente de aceros microaleados.

3) 12:40-13:00: A. García-Escorial, K.R. Cardoso y F.E. Audebert. Aleaciones de aluminio amorfas y nanoestructuradas.

4) 13:00-13:20: R.B. Pérez-Sáez, J.M. San Juan, V. Recarte, M.L. Nó, G. Caruana y O.A. Ruano. Elaboración de aleaciones de $\mathrm{Cu}-\mathrm{Al}-\mathrm{Ni}$ con efecto de memoria de forma mediante pulvimetalurgia.

5) 13:20-13:40: C. García de Andrés, F.G. Caballero y C. Capdevila. Estudio dilatométrico de la descomposición anisotérmica de la perlita en un acero bajo en carbono $(0,11 \mathrm{C}-0,50 \mathrm{Mn})$.

6) 13:40-14:00: A. Martín Meizoso, M. Tellaeche Repáraz y J. Gil-Sevillano. Un generador aleatorio de microestructuras virtuales 3D.

\section{4:00 - 15:00 h: COMIDA \\ 15:00 - 16:00 h: Sesión de paneles y exposición}

16:00 - 17:00 h: CONFERENCIA PLENARIA a cargo de W. Steen (Univ. Liverpool): "Laser material processing: present and future".

Presidente: Rui Vilar (RED CYTED). Secretario: J. J. de Damborenea (CENIM)

17:00 - 18:20 h: Sesión 2 de conferencias (de 15+5 min): RECICLADO A IMPACTO MEDIOAMBIENTAL: Presidente: Federico López Mateos (UCM). Secretario: Félix A. López (CENIM).

1) 17:00-17:20: C. Frías y O. Pérez. Recuperación de ácidos y metales en baños agotados del decapado de aceros inoxidables.

2) 17:20-17:40: A. Cores, A. Formoso, J.L. Verduras y S. Ferreira. Reciclado de aceites residuales siderúrgicos por inyección en toberas del horno alto.

3) 17:40-18:00: A. Chicharro, V. Cala, M.T. Martín y M.T. Larrea. Impacto ambiental por metales pesados en suelos y plantas del entorno de un depósito de chatarra procedente de automóviles de desguace.

4) 18:00-18:20: F.A. López, E. Vila, J.L. Martín de Vidales y A. López-Delgado. Reciclado de las aguas de decapado.

18:20 - 19:20 h: Sesión 3 de conferencias (de 15+5 min): FABRICACIÓN DE ARRABIO Y ACERO

Presidente: Benjamín Fernández (ACERALIA). Secretario: Alejandro Cores (CENIM)

1) 18:20-18:40: L.F. Verdeja, P. Rusek, A. Alfonso y R. González. La temperatura de la intercara arrabio/ refractario como variable determinante de los mecanismos de corrosión del crisol del horno alto.

2) 18:40-19:00: A. Babich, L. García, J. Vega, A. Formoso y A. Cores. Análisis de la operación del horno alto mediante la exergía térmica.

3) 19:00-19:20: J. Jiménez, B. Fernández, J. Sáinz de Ayala, J. Mochón, A. Formoso y F. Bueno. Nuevo modelo matemático para la distribución de carga en el horno alto.

20:00 h Cocktail en el CENIM ofrecido por la CAM (salida del autobús a las 19:30 del Hotel Meliá)

\section{JUEVES 28}

8:45 - 9:30 h CONFERENCIA PLENARIA a cargo de Ignacio Oñate (INASMET): "Ingeniería de superficies. Tendencias y perspectivas de futuro". Presidente: Rafael Naranjo (ACERINOX). Secretario: Sebastián F. Medina (CENIM).

9:30 - 10:30 h: Sesión 4 de conferencias (de 15+5 min): ANÁLISIS Y CALIDAD. Presidente: José $\mathrm{M}^{\mathrm{a}}$ Palacios (SERDEC). Secretario: $\mathrm{M}^{\mathrm{a}}$. T. Dorado (CENIM).

1) 9:30-9:50: J.M. González Diéguez. Nuevos avances en espectrometría de emisión por arco/chispa.

2) 9:50-10:10: A. G. Coedo, M.T. Dorado e I. Padilla. El análisis químico en el control de procesos metalúrgicos. 
3) 10:10-10:30: J. Botella, M.T. Fernández-Collado e I. Fernández de Castillo. Influencia de los elementos residuales cobre, estaño, fósforo y arsénico en el agrietamiento de la superficie del acero inoxidable 18-8 durante la compresión a altas temperaturas.

10:30 - 12:00 h. Café y sesión de paneles y exposición

12:00 - 14:00 h: Sesión 5 de conferencias (de 15+5 min): METALURGIA FÍSICA. Presidente: Santiago Gutiérrez y Sáez de Solabarría (Babcock Wilcox Española). Secretario: José Luis González Carrasco (CENIM).

1) 12:00-12:20: I. Gallego, M.L. Nó y J.M. San Juan. Mecanismos de movilidad de dislocaciones a temperaturas medias en plomo de muy alta pureza (99.9999\%).

2) 12:20-12:40: M.T. Pérez-Prado, J. Ibáñez, M. Morris, M.C. Cristina, O.A. Ruano y G. GonzálezDoncel. Estructura y textura de la fase $\mathrm{Al}_{3} \mathrm{CaZn}$ en una chapa laminada de la aleación superplástica $\mathrm{Al}-5 \% \mathrm{Ca}$ - $5 \% \mathrm{Zn}$.

3) 12:40-13:00: M.M. Petite, I. Gutiérrez, J. Zaitegui y J.I. Larburu. Modelización de la evolución microestructural durante el recocido continuo de chapas de acero bajo en carbono. Predicción de las propiedades mecánicas.

4) 13:00-13:20: A.B. Quispe, S.F. Medina, J.M. Cabrera y J.M. Prado. Estudio de la interacción precipitación-recristalización de un acero microaleado al vanadio con $0,35 \% \mathrm{C}$.

5) 13:20-13:40: F. Gómez, J.M. Gallardo y E.J. Herrera. Algunas observaciones sobre la sinterización del acero austenítico 316L en atmósfera de argón.

6) 13:40-14:00: J.Ma . Amo y J. Durán. Comportamiento a fatiga de uniones a tope en chapas recubiertas soldadas por proceso láser.

\section{4:00 - 15:00 h: COMIDA}

15:00 - 16:00 h: Sesión de paneles y exposición

16:00 - 17:00 h: CONFERENCIA PLENARIA a cargo de Luis Carreras (Grupo TTC) y Javier Anza (Asociación de TT del País Vasco): "Tratamiento térmico en la encrucijada del 2000". Presidente: José Manuel Giral (INASMET). Secretario: Bernardo J. Fernández (CENIM).

17:00 - 18:20 h: Sesión 6 de conferencias (de 15+5 min): CORROSIÓN Y PROTECCIÓN. Presidente: Enrique Otero (Univ. Juan Carlos I). Secretario: Joaquín Simancas (CENIM).

1) 17:00-17:20: A. Aballe, M. Bethencourt, F.J. Botana, M. Marcos y M.A. Rodríguez-Chacón. Seguimiento de la corrosión de la aleación AA2024 en disoluciones de $\mathrm{NaCl}$ mediante la medida del ruido electroquímico.

2) 17:20-17:40: A. Bautista, M. Morcillo, J.A. González, J. Simancas y E. Otero. Comportamiento del aluminio desnudo y anodizado en atmósferas con grados de contaminación muy diferentes.

3) 17:40-18:00: J. López, E. Blanco, J. Botella y L. Esquivias. Caracterización de capas coloreadas sobre AISI 304.
4) 18:00-18:20: A. Conde y J.J. de Damborenea. Corrosión de la aleación 8090 en medios salinos de agresividad moderada.

18:20 - 19:30h: Sesión de paneles y exposición.

21:00 h: CENA DE GALA en el Restaurante Pedro Larumbe, Galería ABC, calle Serrano 61.

\section{VIERNES 29}

8:45 - 10:00 h: CONFERENCIA PLENARIA a cargo de José Luis Martínez (Plan Nacional I+D), José de No (CSIC) y un representante de la Dirección Gral. de Tecnología y Seguridad Industrial (MINER): "Ayudas a la investigación e interrelación industria-investigación". Presidente: José L. Sotelo (UCM). Secretario: Manuel Carsí (CENIM).

10:00 - 11:00 h: Sesión 7 de conferencias (de 15+5 min): METALURGIA NO FÉRREA. Presidente: Antonio Ballester (UCM). Secretario: Francisco J. Alguacil (CENIM).

1) 10:00-10:20: L. Sánchez, A. Roca, M. Cruells y J. Viñals. Estudio de la reactividad de soluciones sólidas jarosita-beudantita por sulfuración-cianuración.

2) 10:20-10:40: F.J. Alguacil. Extraction of As(V) from sulphuric acid solutions by Cyanex 925 .

3) 10:40-11:00: E. Gordo, F. García-Carcedo y J.M. Torralba. Obtención de carburos de tantalio y de niobio por reducción carbotérmica del mineral columbotantalita.

\section{1:00 - 11:30 h: Café}

11:30 - 13:30 h: Sesión 8 de conferencias (de 15+5 min): CORROSIÓN Y PROTECCIÓN. Presidente: Ignacio Fernández del Castillo (ACERINOX). Secretario: Mª.L. Escudero (CENIM).

1) 11:30-11:50: E. Ovejero y J. Toribio. Corrosión bajo tensión de aceros eutectoides con trefilado progresivo.

2) 11:50-12:10: B. Chico, E. Otero, L. Mariaca y M. Morcillo. La corrosión en atmósferas marinas. Efecto de la distancia a la costa..

3) 12:10-12:30: E. Otero, F.J. Pérez, M.P. Hierro, C. Gómez y F. Pedraza. Comportamiento frente a la oxidación a elevada temperatura del acero inoxidable austenítico AISI 304 implantado con baja dosis de cerio.

4) 12:30-12:50: M.C. García Alonso, J.L. González Carrasco y M.L. Escudero. Estudio de la cinética de oxidación de la superaleación MA 956.

5) 12:50-13:10: J.M. Gómez de Salazar, A. Ureña y I. Beneite. Recubrimiento de cobre sobre $\mathrm{Al}_{2} \mathrm{O}_{3}$ mediante reducción autocatalítica.

6) 13:10-13:30: A. Bautista, V. López, E. Otero, R. Lizarbe y J.A. González. Reducción del tiempo y de la temperatura de sellado del aluminio anodizado con aditivos orgánicos.

13:30 - 14:00 h: CLAUSURA 